\title{
Low-rank Properties in the Hybrid Finite Element-Boundary Integral Method
}

\author{
Pieterjan Demarcke $^{1} \quad$ Dries Vande Ginste ${ }^{2} \quad$ Hendrik Rogier $^{3}$
}

\begin{abstract}
The iterative solution of the hybrid finite elementboundary integral equation (FE-BIE) system can be accelerated by using a multilevel fast multipole algorithm (MLFMA) to accelerate the BIE interactions. The far interactions in the MLFMA are of low complexity, which can be traced back to the original dense BIE matrix where off-diagonal submatrices are of low-rank, provided the unknowns are properly ordered. To accelerate the FE part, we investigate the existence of similar low-rank properties in the FE matrix. Previously, they were shown to exist in the FE discretization matrices of elliptic partial differential equations, but not for the non-elliptic time harmonic system of Maxwell's equations. Comparing the Schur complement form of the FE system with a discrete PoincaréSteklov operator, the existence of such low-rank properties is suggested, and this theory is confirmed experimentally by means of a numerical example.
\end{abstract}

\section{INTRODUCTION}

The hybrid FE-BIE method [1] is a widely used approach to numerically solve electromagnetic radiation or scattering problems. It combines the versatility of the FE method to model complex heterogeneous structures with the accuracy and efficiency of the BIE method to solve large homogeneous and potentially unbounded domains. However, the fundamentally different structure of the dense BIE and sparse FE system matrices makes the hybrid FE-BIE system badly conditioned and difficult to solve, both with direct and with iterative methods. When comparing the two techniques, the iterative method seems to be the most scalable approach to solve the hybrid FE-BIE system, provided we find an efficient way to reduce the numerical complexity of one matrix-vector product.

The BIE method can be accelerated considerably by using a multilevel fast multipole algorithm (MLFMA) [2]. For $N_{b}$ boundary unknowns, the algorithm uses a recursive partitioning of the structure to group the unknowns into boxes, and, for the far-field interactions, relies on the plane wave decomposition of the Green's function to replace the $N_{b}^{2}$ direct interactions by a hierarchical set of interactions between boxes. This reduces the computational complexity of one matrix-vector product from $O\left(N_{b}^{2}\right)$ to $O\left(N_{b} \log N_{b}\right)$. The far interactions in the matrix-free MLFMA are of low complexity, which can also be traced back to the original dense BIE matrix, where off-diagonal submatrices are of low-rank, provided the unknowns are properly ordered. Similar to the MLFMA, this lowrank property has a hierarchical structure, which can be used to approximate the dense BIE matrix directly. These low-rank compression techniques have also been proven useful in the MLFMA itself, as demonstrated in [3]. The hierarchical matrix representation can be constructed without the knowledge of the Green's function and its decomposition, and its application to a vector of size $N_{b}$ can typically be performed in $O\left(N_{b}\right)$ or $O\left(N_{b} \log N_{b}\right)$ operations. Examples of such representations are the family of $\mathcal{H}-, \mathcal{H}^{2}$ - and HSSmatrices [4], [5].

Recently, it has become clear that many matrices from discretized systems exhibit some form of lowrank property, and interestingly, this is also true for the sparse system matrices obtained from the discretization of partial differential equations (PDE's), such as the sparse FE matrix. Specifically, it is observed that the off-diagonal blocks of the fill-in after Gaussian elimination of the sparse FE matrix are of low rank. For elliptic PDE's, existence of this low-rank property can be shown theoretically [6], [7]. However, for nonelliptic PDE's such as the time-harmonic system of Maxwell's equations, no such results are available. In this contribution, we provide experimental results regarding the low-rank property in the sparse FE matrix obtained from the discretization of the time-harmonic system of Maxwell's equations. Since the fill-in after Gaussian elimination usually only occurs in direct numerical solvers, special treatment of the sparse FE matrix is needed to make it suitable for approximation by a hierarchical matrix representation. After outlining the general theory in Section 2, we compare the lowrank property of the BIE and FE system matrices by means of a $2 \mathrm{D}$ transverse magnetic (TM) scattering example.

\footnotetext{
${ }^{1}$ Department of Information Technology, Ghent University, Sint-Pietersnieuwstraat 41, B-9000 Gent, Belgium. e-mail: pieterjan. demarcke@intec. UGent.be.

${ }^{2}$ Department of Information Technology, Ghent University, Sint-Pietersnieuwstraat 41, B-9000 Gent, Belgium. e-mail: vdginste@intec.UGent.be.

${ }^{3}$ Department of Information Technology, Ghent University, Sint-Pietersnieuwstraat 41, B-9000 Gent, Belgium. e-mail: hendrik.rogier@intec.UGent.be.
} 


\section{General Formulation}

We consider a homogeneous and isotropic background medium $\Omega_{0}$, characterized by an electric permittivity $\epsilon_{0}$ and a magnetic permeability $\mu_{0}$. Embedded in this medium is a single cylindrical scatterer, aligned along the $z$-axis and of arbitrary cross section $S$ in the $x y$-plane. The potentially inhomogeneous and/or anisotropic scatterer is characterized by the relative permittivity and permeability tensors $\overline{\bar{\epsilon}}_{r}(\boldsymbol{\rho})$ and $\overline{\bar{\mu}}_{r}(\boldsymbol{\rho})$ in each point $\rho \in S$. Using the BIE formulation in the background medium, the radiation condition in the infinite background medium is enforced at the external boundary $C^{+}$of $S$ by providing a direct relation between the unknown tangential electric and magnetic fields on $C^{+}$. Depending on the observed field quantity at $C^{+}$, we differentiate between the electric and magnetic field integral equation (EFIE and MFIE). Focussing on the pure TM case with electric and magnetic fields $\left(\mathrm{e}_{z} \hat{\mathbf{z}}, \mathbf{h}_{t}\right)$, the EFIE and MFIE can be compactly written in operator notation as

$$
\left(\begin{array}{cc}
-\mathcal{K}_{0}+\frac{1}{2} & \mathcal{G}_{0} \\
-\mathcal{J}_{0} & \mathcal{K}_{0}^{\prime}+\frac{1}{2}
\end{array}\right)\left(\begin{array}{c}
\mathrm{e}_{z}^{+} \\
\mathrm{h}_{t}^{+}
\end{array}\right)=\left(\begin{array}{c}
\mathrm{e}_{z}^{\text {in }} \\
\mathrm{h}_{t}^{\text {in }}
\end{array}\right) .
$$

Here, $\mathrm{e}_{z}^{+} \in H^{\frac{1}{2}}\left(C^{+}\right)$and $\mathrm{h}_{t}^{+}=\mathbf{h}_{t} \cdot \hat{\mathbf{t}}^{+} \in H^{-\frac{1}{2}}\left(C^{+}\right)$ are the tangential electric and magnetic field on $C^{+}$, while $\mathrm{e}_{z}^{\text {in }}$ and $\mathrm{h}_{t}^{\text {in }}$ are the tangential components of a time harmonic plane wave of wavenumber $k_{0}$ incident on $C^{+}$. The operator system on the left hand side of (1) represents the exterior Calderón projector, and we refer to [8] for more information about the definition and properties of (1) and the operators $\mathcal{K}_{0}, \mathcal{G}_{0}, \mathcal{J}_{0}$ and $\mathcal{K}_{0}^{\prime}$.

The FE formulation for the electric or magnetic field inside $S$ consists of a weak boundary value problem (BVP) subject to boundary conditions defined on the internal boundary $C^{-}$of $S$. By using Robin-toRobin transmission conditions between $C^{+}$and $C^{-}$, the Cauchy data of the external Calderón projector can be applied as boundary conditions on $C^{-}$, which avoids the problem of spurious solutions at resonance frequencies [8]. The discretization of the FE formulation follows after choosing the appropriate set of curl-conforming test and basis functions defined on a geometrical partitioning (mesh) of $S$. For the $\mathbf{h}_{t}$ field component in $S$, the weak form of the BVP is given by

$$
\begin{array}{r}
\left\langle\nabla_{t} \times \mathbf{w}_{e}, \epsilon_{r, z z}^{-1} \nabla_{t} \times \mathbf{h}_{t}\right\rangle_{S}-k_{0}^{2}\left\langle\mathbf{w}_{e}, \overline{\bar{\mu}}_{r} \cdot \mathbf{h}_{t}\right\rangle_{S} \\
=j k_{0}\left\langle\mathbf{w}_{e}, \mathrm{e}_{z} \hat{\mathbf{z}} \times \hat{\mathbf{n}}^{-}\right\rangle_{C^{-}},
\end{array}
$$

with $\mathbf{w}_{e}$ a curl-conforming test function and $\langle\mathbf{u}, \mathbf{v}\rangle_{S}=$ $\int_{S} \mathbf{u} \cdot \mathbf{v} \mathrm{d} S$ and $\langle\mathbf{u}, \mathbf{v}\rangle_{C^{-}}=\oint_{C^{-}} \mathbf{u} \cdot \mathbf{v} \mathrm{d} C$ the scalar products over $S$ and $C^{-}$, respectively. We assumed that no sources are present in $S$. After discretization of (2), we write the final sparse FE system as

$$
\mathbf{Y}_{\mathbf{H}}\left[\begin{array}{c}
\mathbf{x}_{\mathbf{H}}^{\mathrm{i}} \\
\mathbf{x}_{\mathbf{H}}
\end{array}\right]=\left[\begin{array}{cc}
\mathbf{Y}_{\mathbf{H}}^{\mathrm{ii}} & \mathbf{Y}_{\mathbf{H}}^{\mathrm{i}} \\
\mathbf{Y}_{\mathbf{H}}^{\mathrm{ei}} & \mathbf{Y}_{\mathbf{H}}^{\mathrm{ee}}
\end{array}\right]\left[\begin{array}{c}
\mathbf{x}_{\mathbf{H}}^{\mathrm{i}} \\
\mathbf{x}_{\mathbf{H}}
\end{array}\right]=\left[\begin{array}{c}
\mathbf{0} \\
\mathbf{D} \mathbf{x}_{\mathbf{E}}
\end{array}\right]
$$

with $\mathbf{x}_{\mathbf{H}}^{\mathbf{i}}$ the internal degrees of freedom (DOF) for the magnetic field in $S, \mathbf{x}_{\mathbf{H}}$ and $\mathbf{x}_{\mathbf{E}}$ the DOFs for the tangential magnetic and electric field at $C^{-}$and $\mathbf{D}$ the matrix of inner products between testing and basis functions at $C^{-}$.

\section{Low-rank Properties}

Low-rank properties of dense matrices are revealed by rank-revealing factorizations such as the singular value decomposition (SVD) and the rank-revealing $\mathrm{QR}$ factorization. Using the SVD, a given dense $M \times M$ matrix $\mathbf{A}$ can be represented by the weighted sum of $r \leq M$ rank-1 column matrices $\mathbf{u}, \mathbf{v}$

$$
\mathbf{A}=\sum_{n=1}^{r} \sigma_{n} \mathbf{u}_{n} \mathbf{v}_{n}^{\mathrm{T}}
$$

where both $\mathbf{u}_{n}$ and $\mathbf{v}_{n}$ form an orthonormal set, $n=$ $1, \ldots r$. The singular values $\sigma_{n}$ are ordered to satisfy $\sigma_{r} \geq \sigma_{r-1} \geq \ldots \geq \sigma_{1} \geq 0$. With the Frobenius matrix norm

$$
\|\mathbf{A}\|_{\mathrm{F}}=\sqrt{\sum_{i, j}\left|(\mathbf{A})_{i j}\right|^{2}}
$$

the rank- $k$ approximation $\widetilde{\mathbf{A}}$ of $\mathbf{A}$ that minimizes the error $\|\mathbf{A}-\tilde{\mathbf{A}}\|_{\mathrm{F}}$ is given by

$$
\widetilde{\mathbf{A}}=\sum_{n=1}^{k} \sigma_{n} \mathbf{u}_{n} \mathbf{v}_{n}^{\mathrm{T}},
$$

and the approximation error equals $\sqrt{\sum_{n=k+1}^{r} \sigma_{n}^{2}}$.

\subsection{BIE method}

The integration kernel in the BIE operators of (1) is given by the 2D Green's function $G_{0}\left(\boldsymbol{\rho}, \boldsymbol{\rho}^{\prime}\right)=$ $\frac{j}{4} H_{0}^{(2)}\left(k_{0}\left|\boldsymbol{\rho}-\boldsymbol{\rho}^{\prime}\right|\right)$ with $H_{0}^{(2)}$ the zeroth-order Hankel function of the second kind. From the Hankel addition theorem, it follows that a seperable expansion of the Green's functions exists if the circumscribing circles of the groups of unknowns do not overlap. If the groups are well-separated, the number of required terms in the expansion becomes small, and the corresponding interaction blockmatrix in the discrete operator will be of low rank.

Provided the unknowns are properly ordered, the low complexity of the hierarchical set of far interactions in the MLFMA is reflected in the hierarchical rankstructured form of the BIE matrices $\mathbf{K}_{\mathbf{0}}, \mathbf{J}_{\mathbf{0}}, \mathbf{G}_{\mathbf{0}}$ and $\mathbf{K}_{\mathbf{0}}^{\mathrm{T}}$ corresponding to the operators $\mathcal{K}_{0}, \mathcal{G}_{0}, \mathcal{J}_{0}$ and $\mathcal{K}_{0}^{\prime}$ from (1), respectively. Similar to the MLFMA, a submatrix admissibility criterion based on the distance 
between the interacting unknowns allows the approximation of the dense BIE matrices by a hierarchical matrix structure consisting of inadmissible near interaction blocks and low-rank factorized far interaction blocks, reducing the $O\left(N^{2}\right)$ complexity of the discrete BIE operator to $O(N \log N)$ or $O(N)$.

\subsection{FE method}

The sparse FE system matrix $\mathbf{Y}_{\mathbf{H}}$ can be transformed into a dense matrix by forming the Schur complement to eliminate the interior unknowns $\mathbf{x}_{\mathbf{H}}^{\mathbf{i}}$. As shown in [8], the resulting compressed system matrix corresponds to a discretized Poincaré-Steklov operator. For $S$ formed by a homogeneous medium, this Poincaré-Steklov operator can be expressed equivalenty in terms of operators of the same form as the BIE operators $\mathcal{K}_{1}, \mathcal{G}_{1}, \mathcal{J}_{1}$ and $\mathcal{K}_{1}^{\prime}$ obtained from (1) applied in $S$. The discrete forms of both representations approximate each other when the mesh in $S$ is sufficiently fine [8], and the compressed FE system matrix $\mathbf{Y}_{\mathbf{H}}^{\mathbf{c}}$ will satisfy

$$
\begin{aligned}
\mathbf{Y}_{\mathbf{H}}^{\mathbf{c}} & =\mathbf{Y}_{\mathbf{H}}^{\mathbf{e e}}-\mathbf{Y}_{\mathbf{H}}^{\mathbf{e i}} \mathbf{Y}_{\mathbf{H}}^{\mathrm{ii}}{ }^{-1} \mathbf{Y}_{\mathbf{H}}^{\mathbf{i e}} \\
& =\mathbf{G}_{\mathbf{1}}-\left(\mathbf{K}_{\mathbf{1}}+\frac{1}{2} \mathbf{D}\right) \mathbf{J}_{\mathbf{1}}{ }^{-1}\left(\mathbf{K}_{\mathbf{1}}{ }^{\mathrm{T}}-\frac{1}{2} \mathbf{D}^{\mathrm{T}}\right)
\end{aligned}
$$

Comparing (7) and (8), the origin of the low-rank property observed in the fill-in after Gaussian elimination of the sparse FE matrix is immediately linked to the rank-structured properties of the discrete BIE operators in the same domain. While no analytical expression for the Green's function is available in a general inhomogeneous medium, it is assumed that the integration kernels in the BIE operators remain separable for well separated interactions. It follows that the existence of the low-rank property in the compressed FE matrix will depend on the properties of the discrete operator $\mathbf{J}_{\mathbf{1}}{ }^{-1}$, and as shown in the next Section, simulations suggest that this rank-structured form remains present in $\mathbf{Y}_{\mathbf{H}}^{\mathbf{c}}$.

\section{Numerical EXAMPLE}

We consider a rectangular region $S$ of dimensions $8 \lambda \times L$ formed by the homogeneous material characterized by $\left(\epsilon_{r}, \mu_{r}\right)=(2,1)$, as shown in Figure 1 . By sweeping the length $L$ over the range $\left[\frac{1}{4} \lambda, 16 \lambda\right]$ in increments of $\frac{1}{4} \lambda$, we evaluate the numerical rank of the $M \times M$ submatrix $\mathbf{Z}_{12}$ that describes the interactions between the segment groups $s_{1} \rightarrow s_{M}$ and $s_{\frac{N}{2}+1} \rightarrow s_{\frac{N}{2}+M}$ situated at the left and the right side of the rectangle, respectively. The free-space wavelength $\lambda$ corresponds to an operating frequency of $2.4 \mathrm{GHz}$, and the $M=200$ segments on the left and right sides of the rectangular region are of length $l=\frac{\lambda}{25}$. The total number of boundary segments $N$ is changed to keep

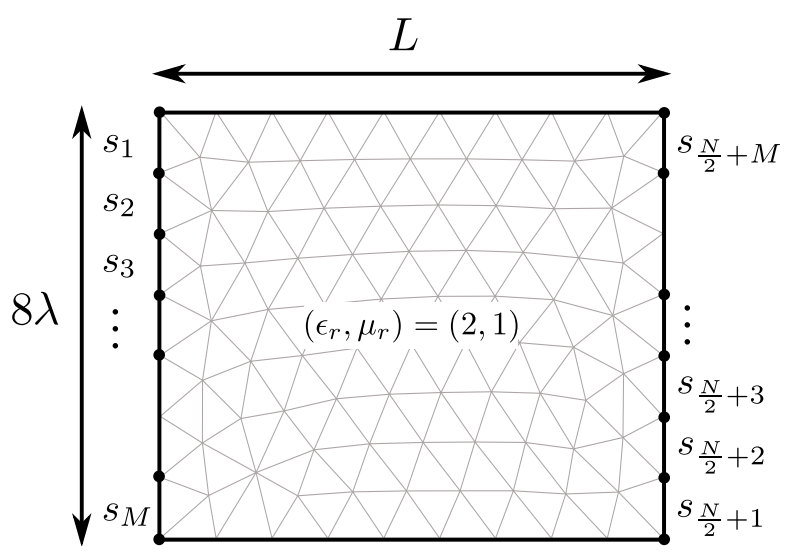

Figure 1. Geometry of the example used to evaluate the low-rank properties in the BIE and compressed FE system matrices.

the segment length as close to $\frac{\lambda}{25}$ as possible while sweeping $L$.

In Figure 2, we compare the numerical rank of the $200 \times 200$ submatrix $\mathbf{Z}_{12}$ as a function of the interaction distance $L$ for the discrete BIE operator $\mathbf{K}_{1}$ and the compressed FE matrix $\mathbf{Y}_{\mathbf{H}}^{\mathbf{c}}$. The desired precision of the low-rank approximation is set to $10^{-6}$, measured using the Frobenius norm (5). To keep the figure apprehensible, the results for the other discrete BIE operators are omitted, but they behave in an almost identical manner to the results for $\mathbf{K}_{\mathbf{1}}$. For small interaction distances up to $L=2 \lambda$, the numerical rank $k$ of the submatrix $\mathbf{Z}_{12}$ decreases according to $k \sim O\left(\frac{1}{L}\right)$, both for the BIE as for the compressed FE system matrix. If objects are scaled in two dimensions, we obtain $k \sim O(1)$, and the complexity of the matrix vector product becomes $O(k N) \sim O(N)$. Once the interaction distance increases beyond $L=6 \lambda$, the results for the discrete FE and BIE operators start to differ. For $\mathbf{K}_{1}$, we observe that the numerical rank of the submatrix $\mathbf{Z}_{12}$ keeps decreasing as a function of $L$. While the size of the dataset is too small to do any reliable trend analysis, the results suggest that $k \sim O\left(\frac{\log L}{L}\right)$, which makes the complexity of the matrix vector product comparable to the $O(N \log N)$ of the MLFMA. For the compressed FE system matrix $\mathbf{Y}_{\mathbf{H}}^{\mathbf{c}}$, it is observed that the numerical rank $k$ of the submatrix $\mathbf{Z}_{12}$ remains almost constant once $L>8 \lambda$. This leads to a complexity similar to the standard $O\left(N^{2}\right)$ matrix vector product complexity, but with a much smaller constant coefficient. We believe that this effect is due to the dispersion error introduced by the finite grid in $S$, which makes the FE approximation of the Poincaré-Steklov operator (7) insufficient to resolve the further decreasing rank beyond a certain interaction distance $L$. At this point, refining the FE mesh is not an option since the object is already large compared to the wavelength, and eventually, the new mesh will 


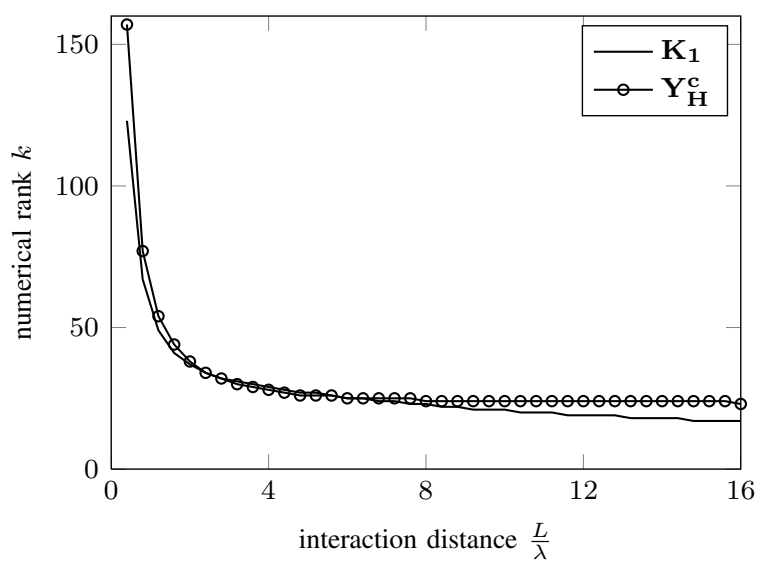

Figure 2. Numerical rank $k$ of the $200 \times 200$ submatrix $\mathbf{Z}_{12}$ of the BIE and compressed FE system matrices $\mathbf{K}_{1}$ and $\mathbf{Y}_{\mathbf{H}}^{\mathbf{c}}$ as a function of interaction distance $L$.

also be insufficient or the number of unknowns will be too large.

Besides the fact that the compressed FE matrix does not appear to be as scalable as the discrete BIE operators, the results show that the iterative solution of the hybrid FE-BIE system can be accelerated up to speeds comparable with the BIE-only MLFMA by using the rank-structured property of the compressed FE matrix. For electrically large objects, this optimal complexity is somewhat reduced, but at this point, grid dispersion and the high number of unknowns will become the limiting factor on the applicability of the FE method.

\section{Conclusion}

We investigated the low-rank property of the FE system matrix obtained after discretization of the nonelliptic time-harmonic system of Maxwell's equations. By comparing the Schur complement form of the FE matrix with a discrete Poincaré-Steklov operator, the connection to the low rank properties of the BIE system matrices was revealed, and the simulations confirmed that the compressed FE matrix has similar rank-structured properties as the BIE matrices. For electrically large objects, the optimal $O(N)$ or $O(N \log N)$ complexity is no longer retained, but it remains considerably lower compared to the standard $O\left(N^{2}\right)$ complexity of a single unaccelerated matrix vector product. Combined with the MLFMA, the hierarchical low-rank approximation of the compressed FE matrix enables the fast iterative solution of the hybrid FE-BIE system up to speeds comparable with the BIEonly MLFMA.

\section{References}

[1] J. L. Volakis, K. Sertel, and B. C. Usner, "Frequency domain hybrid finite element methods for electromagnetics," Synthesis Lectures on Computational Electromagnetics, vol. 1, no. 1, pp. 1-156, 2006.

[2] I. Bogaert, J. Peeters, and F. Olyslager, "A nondirective plane wave MLFMA stable at low frequencies," IEEE Trans. Antennas Propag., vol. 56, no. 12, pp. 3752-3767, 2008.

[3] D. Vande Ginste, E. Michielssen, F. Olyslager, and D. De Zutter, "A high-performance upgrade of the perfectly matched layer multilevel fast multipole algorithm for large planar microwave structures," IEEE Trans. Antennas Propag., vol. 57, no. 6, pp. 1728-1739, June 2009.

[4] W. Hackbusch, B. Khoromskij, and S. Sauter, "On $\mathcal{H}^{2}$. matrices," in Lectures on Applied Mathematics, H.-J. Bungartz, R. H. Hoppe, and C. Zenger, Eds. Springer Berlin, 2000, pp. 9-29.

[5] S. Chandrasekaran, M. Gu, and T. Pals, "A fast $U L V$ decomposition solver for hierarchically semiseparable representations," SIAM Journal on Matrix Analysis and Applications, vol. 28, no. 3, pp. 603-622, 2006

[6] M. Bebendorf and W. Hackbusch, "Existence of $\mathcal{H}$-matrix approximants to the inverse FE-matrix of elliptic operators with $L^{\infty}$-coefficients," Numerische Mathematik, vol. 95, pp. 1-28, 2003.

[7] S. Chandrasekaran, P. Dewilde, M. Gu, and N. Somasunderam, "On the numerical rank of the off-diagonal blocks of schur complements of discretized elliptic PDEs," SIAM Journal on Matrix Analysis and Applications, vol. 31, no. 5, pp. 2261-2290, 2010.

[8] P. Demarcke and H. Rogier, "The poincaré-steklov operator in hybrid finite element-boundary integral equation formulations," IEEE Antennas Wireless Propag. Lett., vol. 10, pp. 503-506, 2011 . 